Florida International University FIU Digital Commons

$12-2-1999$

\title{
Occupational therapists' interests and attitudes toward animal assisted therapy as a treatment modality
}

Lori Susan Buckley

Florida International University

DOI: $10.25148 /$ etd.FI14051868

Follow this and additional works at: https://digitalcommons.fiu.edu/etd

Part of the Occupational Therapy Commons, and the Special Education and Teaching Commons

\section{Recommended Citation}

Buckley, Lori Susan, "Occupational therapists' interests and attitudes toward animal assisted therapy as a treatment modality" (1999). FIU Electronic Theses and Dissertations. 1882.

https://digitalcommons.fiu.edu/etd/1882 


\section{FLORIDA INTERNATIONAL UNIVERSITY}

Miami, Florida

OCCUPATIONAL THERAPISTS' INTERESTS AND ATTITUDES TOWARD ANIMAL ASSISTED THERAPY AS A TREATMENT MODALITY

A thesis submitted in partial fulfillment of the requirements for the degree of MASTER OF SCIENCE

in

OCCUPATIONAL THERAPY

by

Lori Susan Buckley

2000 
To: Dean Ronald Berkman

College of Health Sciences

This thesis, written by Lori Susan Buckley, and entitled Occupational Therapists' interests and attitudes toward animal assisted therapy as a treatment modality, having been approved in respect to style and intellectual content, is referred to you for judgement.

We have read this thesis and recommend that it be approved.

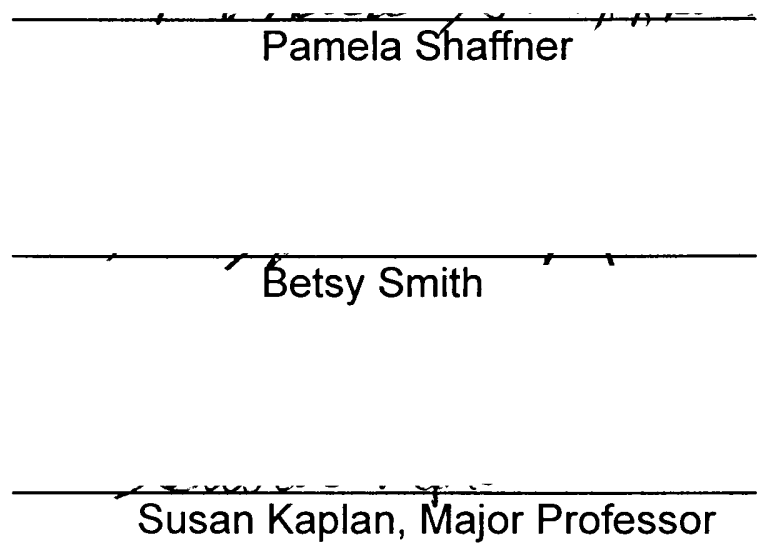

Date of Defense: December 2, 1999

The thesis of Lori Susan Buckley is approved.

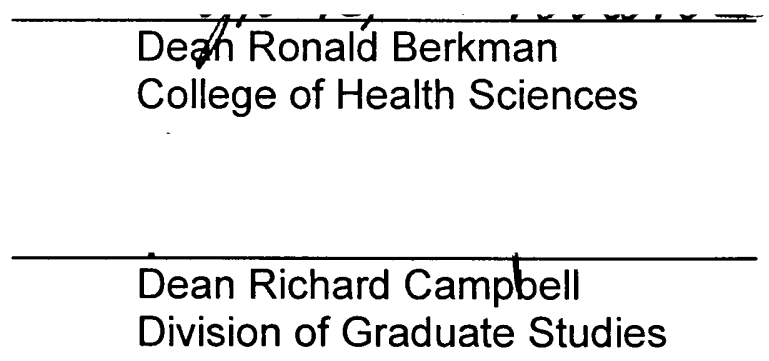

Florida International University, 2000 


\section{ACKNOWLEDGMENTS}

I would like to express my appreciation to the members of my committee

Pamela Shaffner and Betsy Smith, and my chairperson, Susan Kaplan for their support and assistance during the preparation of this work.

I would also like to express my heartfelt thanks to my parents, and my husband, Neil, for their help, support, and encouragement. 
OCCUPATIONAL THERAPISTS' INTERESTS AND ATTITUDES TOWARD ANIMAL ASSISTED THERAPY AS A TREATMENT MODALITY

by

Lori Buckley

Florida International University, 2000

Miami, Florida

Professor Susan Kaplan, Major Professor

One hundred seventy-nine occupational therapists (OTs) responded to a survey regarding their interests and attitudes toward animal assisted therapy as a treatment modality. It was found that most of the practitioners would be interested in learning more about animal assisted therapy and most felt it is a valid treatment modality to be used by occupational therapists. Furthermore, the most common answer to the question "why would you not be interested in participating in an AAT program," was "I feel I don't know enough about it". It was also found that therapists' most commonly selected patient goals for AAT were in the realm of mental health, whereas the least mentioned goals were more physically based goals. Further, a recommendation was made to educate OTs regarding the uses and benefits of AAT through more research, continuing education programs on AAT, publications in professional journals, and media awareness. 


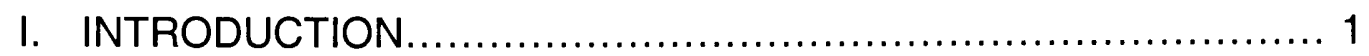

Statement of the Problem .......................................... 3

Objectives and Research Questions ............................ 4

Definitions ...................................................... 5

Assumptions .................................................... 6

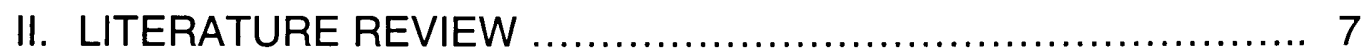

Theoretical perspective ........................................... 7

The health effects of the animal-human bond ..................... 9

The use of animals in the helping professions .................... 10

Animal assisted therapy (AAT) in rehabilitation settings ............ 12

Animal assisted therapy with children ............................. 16

Pet visitation ...................................................... 18

Pet visitation research involving the elderly ..................... 18

Pet visitation research involving psychiatric patients .............. 20

Attitudes of health care workers toward pet visitation programs.... 23

III. RESEARCH PROCEDURES/METHODOLOGY .................... 25

Research questions .......................................... 25

Subjects .................................................... 25

Study design/Statistical analysis ............................... 25

Data collection .................................................. 26

Limitations of the study ....................................... 26

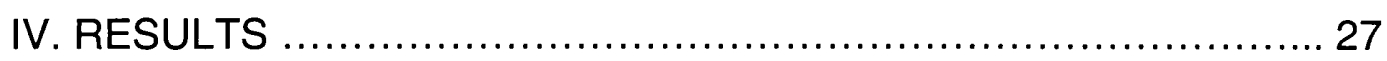

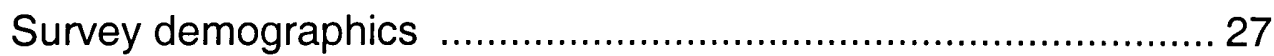

Are OTs interested in learning more about AAT ......................... 31

Is AAT a valid treatment modality to be used by OTs ................. 34

What patient goals does AAT address ........................................ 35

To what extent are OTs involved in AAT programs..................... 36

To what extent are OTs' facilities involved in pet visitation .......... 41

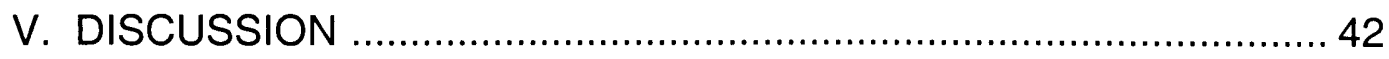

OTs' interest in learning more about AAT ................................... 42

AAT as a treatment modality used by OTs ................................ 42

Patient goals addressed by animal assisted therapy ................. 45 
Interest in participating in AAT programs ................................ 46

To what extent are OTs' facilities involved in pet visitation .......... 47

Summary..................................................................... 47

LIST OF REFERENCES …................................................... 49

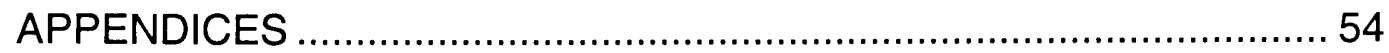




\section{LIST OF TABLES}

TABLE

PAGE

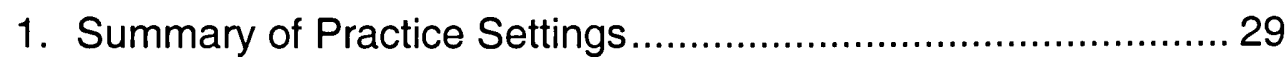

2. Summary of Facilities ..................................................... 30

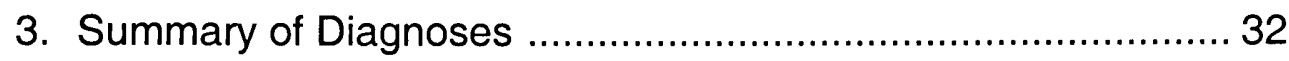

4. Are OTs interested in learning more about AAT .................... 33

5. Do OTs believe AAT is a valid treatment modality ................... 35

6. What patient goals are addressed by AAT .......................... 37

7. Summary of therapists' interest in participating in AAT ........... 38

8. Summary of therapists' reasons for not being interested in participating in an AAT program 39 


\section{Chapter I}

\section{$\underline{\text { Introduction }}$}

Animals have been used for therapeutic purposes throughout history. For instance, the Greeks tried to raise the spirits of chronically ill patients through horseback riding (Hines \& Bustad, 1986). In the $18^{\text {th }}$ century, the York Psychiatric Hospital in England used animals for their calming effects on its mentally disturbed patients (Casey, 1996). Additionally, in the 1800s animals were used in German communities for disabled and disadvantaged people. In the $20^{\text {th }}$ century in Europe, horses were used in rehabilitation and treatment programs for patients with varying diagnoses (Hines \& Bustad, 1986).

Animal assisted therapy (AAT) is the use of animals as a therapeutic modality in order to facilitate the rehabilitation of patients with chronic or acute illnesses. The animals that are used in treatment are appropriately screened and trained to help people with special needs. AAT should be differentiated from animal visitation, which is when animals accompany their owners to a facility to visit the patients. Animal (pet) visitation, also referred to as pet therapy, is primarily used by the nursing profession (Kongable et al., 1989). The main goal of animal visitation is socialization, whereas AAT's main goal is to facilitate patients toward optimum performance. The animals are used in AAT as a treatment modality in goal directed treatment sessions (Bernard, 1995).

Animal assisted therapy is utilized by many professionals, such as occupational therapists, physical therapists, speech therapists, and psychiatrists (Howell-Newman \& Goldman, 1993). Specifically, occupational therapists can 
use an animal such as a dog in order to achieve certain functional goals for the patient. For instance, the goal of increased upper extremity range of motion can be attained through activities such as playing "fetch," using hand signals to command the dog, use of the leash to lead the dog, and petting or brushing the dog. Also, balance can be worked on through patting, brushing, and playing "fetch" with the dog from various positions (e.g. sitting, kneeling, standing, seated on mat). Ambulating with the dog can also attain the goal of mobility. Moreover, the patient's coordination can be improved through brushing and petting the dog, reaching for objects the dog has retrieved, and throwing objects for the dog to fetch. Tone inhibition can also be worked on by patting and brushing the dog to promote relaxation (Hume, 1998).

AAT and animal visitation are used in numerous settings such as nursing homes, psychiatric facilities, acute care hospitals, outpatient facilities, rehabilitation clinics, adult day care centers, Alzheimer day care, and hospices. People with a variety of diseases and disabilities can benefit from animal assisted therapy and pet visitation. Pet visitation has been particularly useful in the treatment of elderly patients in nursing homes. AAT is used successfully in facilitating the rehabilitation of patients with diseases and disabilities such as traumatic brain injuries, spinal cord injuries, cancer, amputation, strokes, multiple sclerosis, visual or hearing impairments, Alzheimer's, dementia, muscular dystrophy, AIDS, developmental disabilities, end stage renal failure, and emotional disturbances (Howell-Newman \& Goldman, 1993). 
Many different species of animals are used in animal assisted therapy, such as fish, birds, rabbits, cats, dogs (Bernard, 1995), sheep, llamas (Johansson, 1998), dolphins (Walker, 1997), and horses (Arkow, 1989). This research project was focused solely on the use of dogs and cats in animal assisted therapy and pet visitation.

Statement of the problem

There are many benefits associated with the use of animals and AAT. For instance, a study by Katcher (1985) found that subjects had decreased blood pressure when petting and talking to a dog, whereas subjects had increased blood pressure when talking to another individual. Another study by Jenkins (1986) concluded that AAT lowers blood pressure levels. His study also found that there is increased sensory stimulation through petting the animal. Further, a study by Brickel (1984) found that participants who were given access to an animal during treatment had greatly reduced depressive symptoms in comparison to a control group. Other studies found that pet therapy improved patients' reality orientation, communication skills, anxiety levels, and concentration (Rowell, 1990).

Another study by Levin and White (Rowell, 1990) found that AAT helped patients' socialization and communication. Additionally, the use of an animal as a treatment modality can often help build rapport between the therapist and the patient (Bernard, 1995). The introduction to the pet provides a topic of conversation and reduces the patient's anxiety as the therapist and patient concentrate on the animal. This interaction helps to establish initial rapport and 
gives the therapist the opportunity to start discussing the goals of therapy as the patient is more willing to communicate (Fick, 1993).

It is also interesting to point out that members of the health care team that work in highly stressful settings such as hospice, ICU, and oncology units find that the animals visits help relieve the tension in their work and also provide comfort for distraught family members (Edwards, 1998).

As one can clearly see, animal assisted therapy offers many benefits. It allows professionals to achieve numerous functional goals, whether it is improved range of motion or strength, increased self worth, or improved communication skills. Despite the numerous benefits, few occupational therapists are utilizing animal assisted therapy. The purpose of this research was to determine the attitudes and interests of occupational therapists in animal assisted therapy as a treatment modality. This study attempted to acquire a better understanding of why this modality is rarely being used by OTs.

\section{Objective and Research Questions}

The objective of this study was to determine the attitudes and interests of occupational therapists toward animal assisted therapy as a treatment modality. The following research questions were addressed:

- Are occupational therapists interested in learning more about animal assisted therapy as a treatment modality?

- Do OTs believe that animal assisted therapy is a valid treatment modality to be used by occupational therapists? 
- What patient goals do occupational therapists think animal assisted therapy addresses?

- To what extent are OTs involved in animal assisted therapy programs?

- To what extent are OTs' facilities involved in pet visitation programs?

\section{$\underline{\text { Definitions }}$}

Animal assisted therapy (AAT) - the use of animals as a therapeutic modality in order to facilitate the rehabilitation of patients with chronic or acute illnesses. The animals that are used in treatment are appropriately screened and trained to help people with special needs. AAT's main goal is to facilitate patients toward optimum performance. The animals are utilized as a treatment modality in goal directed treatment sessions (Bernard, 1995).

Animal/pet visitation (or "Pet Therapy") - when animals accompany their owners to a facility to visit the patients. The main goal of animal visitation is socialization (Bernard, 1995).

Service animal (or companion animal) - an animal that is placed with a person on a full-time basis. Examples include Seeing Eye dogs, hearing dogs for the deaf, assistance animals for the handicapped, or a dog that is owned by an elderly person (Howell-Newman \& Goldman, 1993; lannuzzi \& Rowan, 1990). Therapeutic animal - animal used by the therapist in animal assisted therapy (Howell-Newman \& Goldman, 1993).

Mascot or group pet - animal that lives in the therapeutic setting. This pet provides companionship to the patients as well as the staff (Howell-Newman \& Goldman, 1993). 


\section{Assumptions}

1. It is assumed that survey respondents will provide honest and accurate answers.

2. Animal assisted therapy is a valid and efficacious treatment modality.

3. There are few occupational therapists involved in animal assisted therapy programs. 


\section{Chapter II}

\section{Theoretical perspective}

\section{Literature Review}

The Model of Human Occupation (MOHO) can be used to identify the relationship between an individual and his environment. The Model of Human Occupation was developed by Gary Kielhofner and others (Miller, 1993) as a way to describe occupational behavior. Kielhofner describes occupational behavior as the interaction of a person with his environment. The human occupation system is made up of three subsystems: volitional, habituation, and performance. These subsystems are ordered hierarchically and changes in one system affect other subsystems (Kielhofner \& Burke, 1980).

The volitional subsystem is the highest subsystem and is made up of three aspects: personal causation, interests, and values. The first aspect is a sense of personal causation, which is the notion that an individual has an ability to influence his or her environment. Next, a person's interests are the things that the individual enjoys and chooses to do. The last aspect is values, which are the things that one considers important (Kielhofner \& Burke, 1980).

The middle subsystem is habituation, which involves the organization of behavior into patterns. It consists of habits, which are activities that have become routine through practice. It also comprises roles, which are the internalized expectations of the manner in which one will behave in certain circumstances. This subsystem is governed by the volitional subsystem (Kielhofner \& Burke, 1980). 
The lowest subsystem in the hierarchy is performance. This is comprised of the skills needed in order to produce purposeful behavior. These skills include perceptual motor skills, process skills used to plan and problem solve, and communication skills (Kielhofner \& Burke, 1980). This subsystem is governed by the other two subsystems, and its purpose is to perform the behaviors the other two subsystems call for (Kielhofner \& Burke, 1980).

The Model of Human Occupation can be used as a framework to describe the interaction between the patient and a therapy dog. For example, a patient may not value the companionship of animals and may not have any interest in animals (volitional subsystem). This disinterest in animals disrupts the components of the performance subsystem, as these feelings would affect his or her capability to derive the benefits afforded by animal assisted therapy. If he has no interest in animals, he will either not be included in AAT programs or will not enthusiastically participate in a program. Those patients who do not have an interest in animals will not benefit from AAT; therefore, more traditional modalities of occupational therapy should be employed.

The model also discusses the interaction between a person and his environment. An individual interacts with many different environments, and in doing this, he practices skills and becomes aware of his abilities and limitations (Bruce \& Borg, 1993). Through interaction with an animal, a patient can learn more about his environment and also practice his skills. These skills may include motor skills, perceptual/sensory skills, learning to work with others, concentration, and communication skills. 
The biomechanical model can also be used to explain the use of animal assisted therapy in occupational therapy. The biomechanical model is applied to patients who have "limitations in moving freely, with adequate strength, and in a sustained fashion" (Kielhofner, 1997). These limitations may result from problems in the peripheral nervous system, musculoskeletal system, cardiopulmonary system, or the integumentary system. (Kielhofner, 1997).

This model addresses the capacity for functional motion, which includes joint range of motion, strength, and endurance. Joint range of motion is defined as the potential for a joint to move. Movement is function of the anatomy of the joints and the soft tissue around the joint. Strength involves the ability of a muscle to produce enough tension in order to maintain postural control and move the body parts. Lastly, endurance is the ability to maintain effort over a certain period of time in order to perform a task (Kielhofner, 1997).

A person will experience dysfunction when a limitation in range of motion, strength or endurance interferes with their daily occupational performance. Animal assisted therapy can be used in occupational therapy with patients who have these types of limitation. For instance, the goal of increased range of motion, strength, or endurance can be reached through activities such as playing "fetch," using hand signals to command the dog, use of the leash to lead the dog, and petting or brushing the dog (Hume, 1996). Additionally, in a survey (Casey, 1996) occupational therapist involved in AAT programs indicated that patients could work on increased range of motion and strengthening/exercise through the use of animal assisted therapy. 
The health effects of the animal-human bond

The companionship between humans and animals has shown many benefits. For instance, Jenkins (1986) compared the heart rates and blood pressures of 20 dog owners while petting their dog versus while reading aloud. She found that the pet owners had significantly lower blood pressure while petting versus reading alone. Another researcher (Friedman, 1980) found that pet ownership was positively correlated with 1-year survival of heart patients, with severity of illness and exercise controlled.

Baun et al. (1984) looked at the effects of companion dogs on blood pressure, heart rate, and respiratory rate. The purpose of this study was to see if there was a difference in people's physiological response to petting dogs with which they had established a bond versus dogs with which they were unfamiliar. It was found that petting a dog that one has established a bond with lowers one's blood pressure. Petting a familiar dog produces a relaxing effect.

Serpell (1990) conducted a 10-month prospective study to ascertain the impact of dog or cat ownership on human well-being and health. It was found that pet-owners had a highly significant reduction in minor health problems in the first month of owning their pet. This effect was sustained among dog owners. The pet-owners also exhibited significant improvements in psychological wellbeing in the first 6 months, and this improvement was sustained for the entire 10month period among dog owners. Further, they found that the dog owners improved in self-esteem, and were less fearful of being a victim of crime. This 
study showed the positive effects of pet ownership on human health, and this can, in some circumstances, have long-lasting effects.

In a study at a retirement community, Andrysco (1981) found that five of his patients' self-care activities improved significantly after interacting with a therapy dog. Moreover, other researchers have found that animals offer many psychological benefits such as companionship, love and affection, safety and protection, the incentive to keep busy, reality orientation, and increasing morale (Cusack \& Smith, 1984).

The use of animals in the helping professions

Animals are used in many different ways in the health care field, including service animal programs, residential programs, therapeutic horseback riding, dolphin swim programs, pet visitation programs, and animal assisted therapy. Service animals are used as hearing dogs for the deaf, assistance for the handicapped, and guide dogs for the blind (lannuzzi \& Rowan, 1990).

Animals that are used in residential programs and live within the setting are called mascot pets, which are animals that live in the therapeutic setting (Howell-Newman \& Goldman, 1993). Facilities that these animals reside in include nursing homes, prisons, long-term-care facilities, and psychiatric hospitals (lannuzzi \& Rowan, 1990).

Therapeutic horseback riding is another way that animals are used in the health care profession. It "uses equine-oriented activities for the purpose of contributing positively to the cognitive, physical, emotional, and social well-being 
of people with disabilities" (NAHRA, 1995a). This can serve as an important treatment modality for physical therapists (Iannuzzi \& Rowan, 1990).

Dolphin swim programs give patients the opportunity to interact with dolphins in the wild, with captured dolphins in lagoons, or in aquariums (lannuzzi \& Rowan, 1990). In this type of therapy, dolphins can be used to achieve boals such as increasing attention span, gross and fine motor skills, and speech skills. (Nathanson, 1997).

In pet visitation, animals accompany their owners to a facility to visit the patients (Bernard, 1995). In many cases, weekly visits are made to patients in nursing homes, hospitals, and some private homes (lannuzzi \& Rowan, 1990). The main goal of pet visitation is socialization (Bernard, 1995).

Animal assisted therapy is the use of animals as a therapeutic modality in order to facilitate the rehabilitation of patients with chronic or acute illnesses. The animals are utilized as a treatment modality in goal-directed treatment sessions.

This explanation was used to introduce the reader to the different ways that animals are used in the health care setting. The remainder of this literature review will concentrate mainly on the aspects of animal visitation and pet visitation and how they are utilized in the health care setting.

Animal assisted therapy (AAT) in rehabilitation settings

In performing this literature review, it was quite evident that more research is needed in the field of animal assisted therapy and its use in rehabilitation settings (Casey, 1996; Taylor et al., 1993; Fick, 1993). However, several 
occupational therapists and speech therapists have performed studies in order to gain a better understanding of AAT.

For instance, Fick (1993) performed a study in order to "determine the effect of the presence and absence of a dog on the frequency and types of social interactions among nursing home residents during a socialization group" (p. 529). The sample consisted of 36 residents of a nursing home care unit at a Veterans Administration Medical Center. Subjects were either assigned to social interaction groups with a dog present or absent and the number of social and nonsocial behaviors among the subjects were recorded. It was found that there was a significant difference in verbal interactions among patients when an animal was present. The researcher noted that the results implied that the presence of the dog "provided a comfortable environment that was conducive to the therapeutic goal of facilitating social interactions within the group" (p. 532).

Another study (Taylor et al., 1993) was performed in order to ascertain the effectiveness of a puppy in evoking vocalizations and eye contact among longterm care facility residents. Subjects were either presented with a live puppy or a photograph of a puppy and were asked questions related to what they were seeing. During these sessions, the researcher kept a record of eye contact and vocalizations by the subject. They found that the participants established more eye contact while looking at the live puppy versus looking at the photograph of the puppy. However, this difference was not statistically significant $(p=.1189)$. They also found no significant difference between the vocalizations evoked by the live puppy versus the photograph of a puppy. However, they did find that the 
total time spent with the live puppy was significantly longer than the time spent with the photograph. These researchers suggested that more rigorous studies be done to look at the relationship between animals and humans.

A survey was performed by Casey (1996) in order to describe how occupational therapists use animal assisted therapy, and their opinions about the value of this treatment modality. Ninety questionnaires were sent out to occupational therapists who had used AAT with their patients at some time. Due to a low response rate, the researcher was only able to use $23(26 \%)$ of the total number of surveys in her study. It was found that dogs were by far the most commonly used animals in AAT treatment (83\%). Other animals that were used included cats, rabbits, birds, puppies, kittens, monkeys, ducks, and guinea pigs/hamsters.

Additionally, occupational therapists indicated the types of patients that benefit from AAT: primarily cerebrovascular accident (CVA), traumatic brain injury (TBI), spinal cord injuries (SCI), orthopedic conditions, dementia, and psychiatric patients. It was also found that through AAT, patients could work on the following goals: increased range of motion, increased socialization/ participation, strengthening/exercise, speech/communication skills, improved affect, and sensory/tactile stimulation. The researcher also asked the OTs about the drawbacks that they might have found while using AAT. The most common response was, "some patients are scared of dogs" (Casey, 1996). Other responses included allergies and sharp claws. The researcher stated in 
conclusion that, "PFT [Pet Facilitated Therapy] has tremendous promise of being a better recognized modality in occupational therapy" (Casey, 1996).

Animal assisted therapy can also be used in the psychiatric setting. Holcomb and Meacham (1989) performed a retrospective study in order to compare patient attendance to occupational therapy groups that used AAT with their attendance to other types of occupational therapy groups. Isolated patients were concentrated on in this study. The researchers defined an isolated patient as, "those patients [who] ... rarely communicate with others and more frequently fail to take advantage of various voluntary groups offered on the unit" (Holcomb and Meacham, 1989, p.259). The OT groups that were offered in addition to the AAT group included craft, living skills, exercise, discussion, communication, assertiveness, and chemical dependency groups. The AAT group, termed the "Hug-a-Pet" group, was open to all inpatients, and brought the patients into therapeutic contact with animals for about one hour.

Data was gathered for two years at Hennepin County Medical Center's inpatient psychiatric unit. The researchers found that the AAT group had a significant effect on patient attendance with patients attending AAT more frequently than all other OT groups. Additionally, the Hug-A-Pet group attracted more isolated patients than any other OT group $(p<.01)$. The researchers concluded that AAT provides an opportunity for isolated patients to become more socially interactive. Also, they consider it an "excellent vehicle for clinical assessment and diagnosis for all patients, especially for isolated patients" (Holcomb and Meacham, 1989). 
Animal assisted therapy has also been reported to be effective in its use in speech therapy. A case study (Adams, 1997) was done on a 72-year-old female who had experienced two cerebral vascular accidents (CVAs), along with other medical complications. Two therapy dogs were used in order to achieve the therapist's treatment goals, such as increasing word initiation and verbalization behaviors. After eight treatment sessions with the animals, they found that the patient increased one-word answers and more frequently identified certain objects. Her verbalization behaviors also increased as a result of the treatment sessions involving the dogs (Adams, 1997).

Animal assisted therapy with children

Several studies have been performed to look at the effects of animal assisted therapy on children. For instance, Redefer and Goodman (1989) did a study to investigate the effects of therapy dogs on autistic-like, developmentally delayed children. There were 12 subjects in this study, ranging from five to 10 years in age. All of the patients were notably disordered, functioned in the mentally retarded range, and displayed autistic features such as social withdrawal, unusual or absent language, and idiosyncratic habits. The subjects were given eight sessions of animal assisted therapy with a psychotherapist. The researchers found a highly significant increase in prosocial behavior $(p<.01)$, and a decrease in self-absorption among the children. They also noted that the children displayed fewer autistic behaviors, such as humming noises, spinning objects, and roaming. Additionally, the children displayed more socially 
appropriate behaviors such as joining the therapist in playing games, reaching up for hugs, and imitating the therapist's actions (Redefer \& Goodman, 1989).

Gonski (1985) did a study to illustrate the effects of animal assisted therapy on adolescent males in a residential setting. In several case studies, she found that the use of dogs as her therapeutic assistant facilitated her relationship with her adolescent clients. She found, in conclusion, that well-trained carefully chosen dogs could be used successfully in psychotherapy with children.

A case study was performed by Granger et al. (1998) to determine the effects of animal assisted therapy on two emotionally disturbed children. These two children, aged 11 and 12, participated in approximately 12 AAT sessions. Goals for the participants were set by each student's special education teacher. Examples of goals included: decreasing negative comments, decreasing distractibility, increased eye contact with people, increased sense of control over environment, and the improvement of the appropriateness of voice tone with people. The researchers found that the first participant in the study decreased his negative verbal statements and increased his positive verbal statements to others. He also displayed better patience and voice tone. The second participant improved with his problem solving skills (Granger et al., 1998).

Heindl (1996) performed a study to determine if AAT could be used to decrease behavior problems and increase self-concept in children who were seriously emotionally disturbed. The researchers used a randomized controlgroup design wherein the experimental group participated in an AAT program once a week for 6 weeks. The control group participated in the traditional 
therapeutic activities. They found that after the AAT program, the children in the experimental group showed a significant decrease in problem behaviors. These children also displayed a non-significant difference in self-concept, as compared to the control group (Heindl, 1996).

\section{Pet Visitation}

Pet visitation research involving the elderly

Several studies have been done in order to look at the effects of pet visitation on the elderly. For example, Brickel (1979) explored the therapeutic effects of cat mascots on hospital ward staff. In this study, the subjects were the nursing staff of a hospital unit in which cat mascots had been present for two years. The nursing staff was interviewed regarding interactions between the patients and the animals and other questions concerning the presence of the cat mascots. The nursing staff reported that the patients' interest in the cats varied depending on their interest in pets, mobility, and the degree of their disorder. It was estimated that $50-80 \%$ of the patients enjoyed the company of the pets if they happened to be nearby. Additionally, some staff members reported that the presence of the pets led patients to interact with other patients or staff. It was also stated that the pets helped keep patients in touch with reality. Overall, the researcher stated that "the available evidence indicates that implementation of pet mascots may prove significant in facilitating such therapeutic processes" (Brickel, 1979, p. 372).

Perelle and Granville (1998) studied the effects of a pet visitation program on the social behavior of nursing home residents. Fifty-three subjects, with a 
mean age of 75.4 years, participated in this study. The researchers developed a Patient Social Behavior Scale as the evaluating instrument. Using this scale, the nursing staff evaluated patients' social and self-maintenance behaviors. It was found that there was an immediate positive change in patients' behaviors, and this change was maintained for at least one month after the termination of the study.

Harris et al. (1993) investigated the effects of a pet visitation program on the homebound elderly. The sample consisted of 16 homebound patients who were between the ages of 65 to 91 years. The subjects' vital signs were taken before and after each control and experimental visit. During the first four weeks (the control period) they were visited by a nurse. During the experimental period, they were visited by the nurse, the pet, and the pet's owner. In analyzing the data, researchers found that the subjects' blood pressure was lowered significantly more in the experimental period than in the control period.

Additionally, pulse rate was lowered in both kinds of visits. Their respiratory rate increased with the control period, but did not increase with pet visitation. Half of the subjects completed a General Well-Being Scale and they found no differences between the experimental and control groups for this factor. With regard to this finding, the researchers stated that the tool they used might not have been sensitive enough to measure any differences (Harris et al., 1993).

Robb et al. (1980) conducted a study to determine how effective certain objects were as catalysts for social behavior. She presented a wine bottle, a plant, and a caged puppy to the elderly residents of a veteran's administration 
hospital. She wished to see which objects would more positively influence the patients' social behaviors, such as smiling, looking at object, verbalizing, and leaning toward the stimulus. She found that the caged puppy induced the biggest change among patients' social behavior. Moreover, she found that the patients' verbalizations became more social in nature (Robb, 1980).

Robb (1981) did another study to ascertain the costs and benefits of pet visitation and to determine the perceptions of nurses about dogs in their facility. The study design involved a control group, an experimental/no-pet group, and an experimental/pet group. She found no differences between the three groups on numerous psychological factors; she attributed this to the baseline mental status of the patients. She did find, however, that the nursing staff reported that the patients who were disoriented and confused benefited from the pet visits.

Moreover, after the institution of the pet visitation, the nurses' opinions of the dog in the facility changed from "generally neutral" to "generally favorable." The nurses also remained that they did not think that the use of pet visitation would decrease their workload (Robb, 1981).

\section{Pet visitation research involving psychiatric patients}

Many studies have been done in order to look at the effects of pet therapy among psychiatric patients. For instance, Zisselman et al. (1996) performed a pilot study at a geriatric psychiatry unit. This study examined patients' changes in self-care functioning, disoriented behavior, depressive mood, irritable behavior, and withdrawn behavior in response to a pet visitation program versus an exercise program. It was found that patients who received the pet visitation 
program exhibited less irritable behavior than patients who went through the exercise program $(p<.07)$. Also, this study found that women with dementia had significantly improved irritable behaviors with either pet therapy or exercise intervention $(p<.02)$. They found improvements in self-care functioning and withdrawn behavior among patients receiving the pet visitation program although these changes were not statistically significant.

In another study, Kongable et al. (1989) concluded that the presence of a dog increased the number of social behaviors among patients with Alzheimer's Disease. An additional study by Kongable et al. (1990) investigated the perceptions of a pet visitation program among nursing staff in an Alzheimer's unit. Generally, it was found that the staff thought pet therapy could be a valuable intervention for patients in a health care setting. Staff members mentioned the benefits of pet therapy: increased self-esteem, heightened sense of control over one's environment, socialization, sensory stimulation, and relaxation effects. Nursing staff also indicated several risks associated with pet therapy. These risks included hazard to the dog or patient, dislike of the pet, sensory overload, and the responsibility that was placed on the nursing staff.

Walsh et al. (1995) conducted a study to determine the effect of pet therapy on dementia patients. Subjects in this study were either assigned to an experimental group where they received pet therapy or a control group where they did not receive pet therapy. The researchers found that the subjects in the experimental group experienced a significant improvement in heart rate in contrast to the control group. They also found no statistically significant 
difference in blood pressure between the two groups. Moreover, they indicated that there was a substantial drop in the noise level among the experimental group versus the control group.

In another study investigating the effects of pet visitation on psychiatric patients, Beck et al. (1986) found that patients more frequently attended psychiatric groups in which birds were present. Moreover, they found that patients attending such groups exhibited significantly less hostile behavior than the patients in groups with no animals present.

Thompson (1981) examined the behavior changes among elderly psychiatric patients and set guidelines for the implementation of pet visitation programs in institutions. The study's design involved a control group who did not interact with animals and an experimental group who participated in a pet visitation program for 6 weeks. They found that the "patients with moderate functional impairment improved significantly over both the control subjects and pet therapy subjects on extremes of the range (low impairment and high impairment)" (Thompson, 1981). The researchers note that this indicates that professionals must be selective in choosing patients for pet visitation programs (Thompson, 1981).

Limond et al. (1997) conducted a study to determine the effects of a pet on the behavior of children with severe learning disabilities. Eight children with Down's syndrome participated in sessions with a real dog, an imitation dog, and an adult who was the animal's handler. During each session, the child was visited by a real dog for seven minutes, then an imitation dog for seven minutes. 
The imitation dog was a toy dog that was similar to the real dog in size, texture, and color. They found that the children were more attentive to the real dog, and were more responsive to the adult who was accompanied by the real dog. The children also displayed significantly more verbal and non-verbal initiation with the real dog present (Limond et al., 1997).

Attitudes of health care workers toward pet visitation programs

Crowly-Robinson \& Blackshaw (1998) studied nursing staff members' attitudes toward a pet visitation program in a Veteran's home. Questionnaires were given to the staff members regarding the staff's attitudes toward pets in general, and their attitudes toward a pet visitation program. They found that over $85 \%$ of the respondents indicated that they liked dogs. Thirty-four $(72.3 \%)$ of the respondents reported that they were in favor of a pet visitation program being put in place, and $63.8 \%$ thought it would work. They found that staff members who liked dogs favor having a pet visitation program more than those who did not like dogs. Moreover, most of the staff $(74.5 \%)$ did not think that the pet visitation program would increase their workload, and $6.4 \%$ felt it would decrease their workload. The researchers felt that it would be beneficial to administer such a survey in any facility that is considering the implementation a pet visitation program, as it would allow staff participation (Crowly-Robinson \& Blackshaw, 1998).

Kranz and Schaaf (1989) performed a study to ascertain nursing staff members' attitudes toward a pet visitation program before and after the introduction of the program. Overall, they found that staff members were 
favorably inclined to a pet program. However, they found that many were concerned about possible risks to the staff and the patients. The risks that were mentioned included allergic reactions, the introduction of fleas and ticks to the facility, and pet-transmitted diseases. After the inception of the pet visitation program, the staff's positive attitudes increased slightly. This was reflected by a decrease in their worries about the possible risks associated with the program. 
Chapter III

\section{Methodology}

$\underline{\text { Research Procedures/Methodology }}$

\section{$\underline{\text { Research questions }}$}

- Are occupational therapists interested in learning more about animal assisted therapy as a treatment modality?

- Do OTs believe that animal assisted therapy is a valid treatment modality to be used by occupational therapists?

- What patient goals do occupational therapists think animal assisted therapy addresses?

- To what extent are OTs involved in animal assisted therapy programs?

- To what extent are OTs' facilities involved in pet visitation programs? $\underline{\text { Subjects }}$

Surveys were sent out to 300 occupational therapists (OTRs) practicing in physical disabilities, gerontology, mental health, and pediatrics. Names were obtained from a nationwide randomly selected mailing list through the American Occupational Therapy Association (AOTA).

Study design/Statistical analysis

The data was collected using an original questionnaire. The first part of the questionnaire was comprised of questions regarding demographics and rendered continuous and categorical data. The second part of the questionnaire relates to animal assisted therapy and pet visitation. This section includes multiple choice 
questions as well as Likert scales. A section for comments, which was optional, was included at the end of the questionnaire.

The study design was descriptive research using percentages and frequencies to summarize single variables. Cross tabulations were also done to look at relationships between pairs of variables. Counts, percentages, means, and ranges were used to analyze the demographic data and the responses given on the Likert scales. The SPSS statistical analysis program was used to calculate the data.

\section{Data collection}

First, a pilot study was done on eight occupational therapists practicing in Florida. Then, after appropriate revisions 300 surveys were sent out to occupational therapists that were randomly selected by the American Occupational Therapy Association. All answers remained confidential.

\section{Limitations of the study}

1. Volunteer bias may be present.

2. Only OTRs that are members of the American Occupational Therapy Association were surveyed. This might have affected the generalizability of the study. 


\section{Chapter IV}

\section{$\underline{\text { Results }}$}

The results of this study are reviewed in 5 sections. The first section reports demographic data. The next 4 sections contain the results obtained from the research questions. The results from this study are based on percentages, frequencies, and cross tabulations that were obtained from the survey responses and calculated utilizing the SPSS statistical program.

\section{Survey Demographics}

One hundred seventy-nine surveys (59.7\%) were returned by occupational therapists. One hundred sixty-seven (93.3\%) of the respondents were female, and twelve $(6.7 \%)$ were male. The ages of the respondents ranged from 23 to 75 , with a mean of 37 . One hundred twenty-four $(69.3 \%)$ respondents indicated that a bachelor's degree was the highest educational level achieved, followed by twenty-five (14.0\%) practitioners with entry level master's degrees, and fifteen $(8.4 \%)$ with a master's degree in another field. Seven $(3.9 \%)$ of the respondents indicated that they had an advanced master's degree in occupational therapy, five therapists $(2.8 \%)$ obtained a certificate in occupational therapy, and one person indicated that she had received her doctorate in another field. Two practitioners indicated that their highest level of education was other than those listed; this included an associate's degree in occupational therapy and a therapist finishing her doctorate in social policy and education.

Survey results indicated that the mean years of experience as an occupational therapist was four. More specifically, forty-seven $(26.3 \%)$ of the 
respondents have had seventeen or more years of experience as an occupational therapist, thirty-nine (21.8\%) therapists had three to six years experience, and thirty-two (17.9\%) have had one to two years of experience. Moreover, thirty-one (17.3\%) respondents have had eleven to sixteen years of experience, twenty-four (13.4\%) indicated they have had seven to ten years of experience, and six (3.4\%) reported that they had less than one year of experience.

The returned surveys represented the five regions of the United States, with $27.2 \%$ returned from the northeast, $21.3 \%$ from north central, $20.7 \%$ from the southeast, $18.3 \%$ from the west, and $12.4 \%$ returned from the south central region.

Seven (3.9\%) respondents indicated that they are not currently practicing. The remainder of the respondents $(96.1 \%)$ reported that they are currently practicing. Survey results also indicated that $47(26.3 \%)$ of the respondents' current area of primary practice was in physical disabilities, followed by 31 (17.3\%) therapists working in gerontology. It was also found that $23(12.8 \%)$ of the therapists worked in the school system, and $23(12.8 \%)$ also worked in developmental disabilities. Please see Table 1 for a summary of practice settings.

Of the 176 respondents to the question regarding the facility at which the therapist currently works, $32(17.9 \%)$ indicated that they work at a skilled nursing facility or an intermediate care facility. Twenty-four (13.4\%) respondents indicated that they work in a general hospital in areas other than a rehabilitation 
unit, and $24(13.4 \%)$ work in a school system setting. Moreover, $17(9.5 \%)$ work in a rehabilitation center or hospital. "Other facilities" listed included a state agency, and an OTA program. Several respondents indicated that they work at several different facilities. Please see Table 2 for a summary of facilities.

Table 1

\section{Summary of Practice Settings}

$(n=178)$

Practice settings Frequency Percent of respondents

Physical disabilities 47 26.3

Gerontology 31 17.3

School system 23

12.8

Developmental disabilities

Other pediatric areas

Mental health

10

5.6

Hand Therapy

8

4.5

Education

7

3.9

Home health

5

2.8

Administration / management

4

2.2

Work hardening

2

1.1

Other

.6 
Table 2

Summary of facilities

$(n=176)$

Facility

Skilled nursing facility Frequency Percent of Respondents

School system

General hospital - other areas

24

13.4

Rehabilitation center/hospital

Outpatient clinic

General hospital - rehab unit

Private practice

Psychiatric hospital

University / College

Early intervention program

Home health agency

Other

Pediatric hospital

Independent living center, Group home,

or residential care

Day care program

Sheltered workshop

VA medical center

Public health agency 
When answering the survey question regarding how many years the therapists had been working at their current facility, $50(27.9 \%)$ of the 176 total respondents indicated that they had been working for 1-2 years at their current facility. Forty-four of the respondents $(24.6 \%)$ had been working for 3-6 years at their facility, and $39(21.8 \%)$ had been working at their current facility for less than 1 year. Moreover, $18(10.1 \%)$ of the respondents had been working at their facility for $7-10$ years, and $15(8.4 \%)$ indicated that they had $11-16$ years of experience at their current facility. Lastly, $10(5.6 \%)$ of the respondents reported that they have worked more than 17 years at their current facility.

Respondents indicated that the most common diagnoses seen at their facility were orthopedic problems (64.8\%), cerebrovascular accidents (55.3\%), arthritis $(50.3 \%)$, developmental disabilities $(40.2 \%)$, traumatic brain injury (40.2\%), visual problems (36.3\%), and dementia (34.1\%). Please see table 3 for a summary of diagnoses.

Research question 1: Are occupational therapists interested in learning more about animal assisted therapy as a treatment modality?

Eighty (44.7\%) practitioners indicated that they agree with the statement regarding their interest in learning more about animal assisted therapy as a treatment modality on survey question number 19. Forty-five therapists reported that they neither agreed nor disagreed to the statement. Furthermore, 40 therapists indicated that they strongly agreed with the statement. Please see table 4 for a summary of answers to question number 19. 
Table 3

Summary of diagnoses

( $n=177)$

\begin{tabular}{lrr}
\hline Diagnosis & Frequency & $\begin{array}{r}\text { Percent of } \\
\text { respondents }\end{array}$ \\
\hline Orthopedic problems & 116 & 64.8 \\
Cerebrovascular accident (CVA) & 99 & 55.3 \\
Arthritis & 90 & 50.3 \\
Developmental disabilities & 72 & 40.2 \\
Traumatic brain injury & 72 & 40.2 \\
Visual problems & 65 & 36.3 \\
Dementia & 61 & 34.1 \\
Parkinson's disease & 59 & 33.0 \\
Cerebral Palsy & 59 & 33.0 \\
Cancer & 56 & 31.3 \\
Amputations & 55 & 30.7 \\
Mental impairments & 49 & 27.4 \\
Autism & 48 & 26.8 \\
Alzheimer's disease & 47 & 26.3 \\
Depression & 46 & 25.7 \\
Hearing problems & 46 & 25.7 \\
Spinal cord injuries & 40 & 22.3 \\
Multiple Sclerosis & 33 & 18.4 \\
Spina Bifida & 24 & 13.4 \\
Muscular Dystrophy & 19 & 10.3 \\
Schizophrenia & 17 & 9.5 \\
AlDS & 10 & 5.6 \\
\hline
\end{tabular}


Table 4

Are occupational therapists interested in learning more about AAT

$(n=178)$

Frequency Percent of respondents

\begin{tabular}{lcc}
\hline Agree & 80 & 44.7 \\
Neither agree nor disagree & 45 & 25.1 \\
Strongly agree & 40 & 22.3 \\
Disagree & 9 & 5.0 \\
Strongly disagree & 4 & 2.2 \\
\hline
\end{tabular}

Chi-square tests were performed on certain pairs of questions that were considered to be particularly important to the study. A chi-square test was performed to determine the relationship between therapists' enjoyment of animals (question 12) and whether they would be interested in learning more about AAT (question 19). Significance was found at $X^{2}(1)=8.97, p<.003$ and it was found that seventy-two percent $(n=107)$ of the therapists who indicated they strongly agree or agreed with the statement that they enjoyed the company of animals also stated they were interested in learning more about AAT (strongly agreed or agreed). Moreover, fifty-seven percent $(n=16)$ of respondents who stated they did not enjoy the company of animals indicated that they were not interested in learning more about AAT (neither agreed nor disagreed, disagreed, or strongly disagreed). 
A chi-square test was also done to look at the relationship between the respondents' age and whether they would be interested in learning more about AAT. Significance was found $X^{2}(4)=11.58, p<.021$, and it was found that eightytwo percent $(n=46)$ of respondents age twenty to twenty-nine indicated that they strongly agreed or agreed with the statement that they were interested in learning more about AAT.

Research question 2: Do OTs believe that AAT is a valid treatment modality to be used by occupational therapists?

Sixty-three $(35.2 \%)$ practitioners indicated that they agree with the statement that AAT is a valid treatment modality to be used by OTs. Forty-four $(24.6 \%)$ therapists reported that they strongly agreed to the statement.

Furthermore, $39(21.8 \%)$ therapists indicated that they neither agreed nor disagreed with the statement. Please see table 5 for a summary of answers to this question.

A chi-square test was performed to determine a relationship between respondents' enjoyment of animals and whether they feel AAT is a valid treatment modality. Significance was found at $X^{2}(2)=12.87, p<.002$, as sixty-five percent of the respondents $(n=97)$ who indicated they strongly agreed or agreed with the statement that they enjoyed the company of animals stated that they strongly agreed or agreed that AAT is a valid treatment modality. However, fiftyseven percent $(n=16)$ who indicated that they did not enjoy the company of animals stated that they did not agree with the statement that AAT is a valid 
treatment modality to be used by OTs (neither agreed nor disagreed, disagreed, or strongly disagreed).

Table 5

Do occupational therapists believe AAT is a valid treatment modality to be used by OTs?

$(n=179)$

\begin{tabular}{lrr}
\hline & Frequency & $\begin{array}{r}\text { Percent of } \\
\text { respondents }\end{array}$ \\
\hline Strongly agree & 44 & 24.6 \\
Agree & 63 & 35.2 \\
Neither agree nor disagree & 39 & 21.8 \\
I don't know & 18 & 10.1 \\
Disagree & 11 & 6.1 \\
Strongly disagree & 2 & 1.1 \\
\hline
\end{tabular}

Research question 3: What patient goals do occupational therapists think animal assisted therapy addresses?

Survey question 21 looked at what patient goals OTs felt that AAT addresses. The most common selected goals included: increase social interaction $(90.5 \%)$, sensory stimulation $(88.8 \%)$, increase the display of emotions (83.2\%), decrease depressive symptoms (82.1\%), decrease anxiety (74.9\%), speech/communication skills $(73.2 \%)$, and facilitate patient/therapist interaction (66.5\%). Other goals included increase range of motion $(63.1 \%)$, increase concentration (54.2\%), and increase reality orientation (54.2\%). Other 
goals that were listed included, decrease blood pressure and heart rate, increase attention span, eye contact, interaction, independence, tone, and standing tolerance. Additionally, several OTs responded similarly to this, "all of the above could apply depending on how creative the therapist is and how the animal is used with the patient..." The data are summarized in table 6.

Research question 4: to what extent are OTs involved in animal assisted therapy programs?

Survey question number 16 inquired as to whether the practitioners had ever participated in an AAT program. One hundred forty-one (78.8\%) respondents indicated that they had never participated in an AAT program, whereas 35 (19.6\%) reported that they had. Moreover, question number 15 asked whether their facility currently has an AAT program in place using cats and dogs. A majority of the respondents $(131 ; 73.2 \%)$ indicated that their facility does not have a program in place. Twenty $(11.2 \%)$ of the therapists indicated that their facility did have an AAT program in place.

The therapists were also asked, if they had never participated in an AAT program, would they be interested in participating in one. Among the 141 practitioners who responded to this question, $53(29.6 \%)$ indicated that they might if they had more information about it. Twenty-eight (15.6\%) practitioners reported that they probably would, and $24(13.4 \%)$ therapists said that they would definitely participate in an AAT program. Please see table 7 for a summary of responses to question number 17. 
Table 6

What patient goals are addressed by animal assisted therapy

$(n=177)$

Patient goal

Frequency Percent of respondents

Increase social interaction

162

90.5

Sensory stimulation

159

88.8

Increase display of emotions

149

83.2

Decrease depressive symptoms

147

82.1

Decrease anxiety

134

74.9

Speech/communication skills

131

73.2

Facilitate patient/therapist interaction

119

66.5

Increase range of motion

113

63.1

Increase concentration

97

54.2

Increase reality orientation

97

54.2

Increase balance

95

53.1

Strengthening

77

43.0

Decrease tone

69

38.5

None of the above goals

4

2.2

I haven't heard of AAT

7

3.9 
Table 7

Summary of therapists' interest in participating in an AAT program

$(n=141)$

\begin{tabular}{lcr}
\hline Interest & Frequency & Percent of respondents \\
\hline Yes, definitely & 24 & 13.4 \\
Probably & 28 & 15.6 \\
I might if I had more information & 53 & 29.6 \\
Probably not & 23 & 12.8 \\
No & 13 & 7.3 \\
\hline Total & 141 & 78.8 \\
\hline
\end{tabular}

In question number 18, therapists were asked why they would not be interested in participating in an AAT program. The most common responses were, "I feel I don't know enough about it" (26.3\%), reimbursement issues (17.3\%), "I don't have the time" (15.1\%), and "I'm concerned about cleanliness." Please see table 8 for a summary of therapists' reasons for not being interested in participating in an AAT program. Other responses included fleas, patients may be allergic, and AAT would not be appropriate in their particular setting (examples - Neonatal Intensive Care Unit, academic/university setting, industrial rehabilitation, and outpatient/hands). Additionally, several practitioners responded similarly to this, "I feel there are more important issues to work on. I 
know they enjoy pets, but reimbursement for this may not be considered direct service [according to the current Medicare regulations]."

Table 8

Summary of therapists' reasons for not being interested in participating in an AAT program

$(n=118)$

Reason

Frequency

Percent

I feel I don't know enough about it

Reimbursement issues

31

17.3

I don't have the time

I'm concerned about cleanliness

I'm concerned about the possible legal issues

I am allergic

I'm concerned about the safety of the patients

I'm concerned about pet transmitted diseases

I'm concerned about the safety of the animals

I don't want to work with animals

I am interested but my facility doesn't support it

8

I don't think the patients would want to work with animals

I don't believe AAT is effective

Upon performing a chi-square test, it was found that there was a significant difference in the percentage of respondents who said they would be 
willing to participate in an AAT program by their enjoyment of animals, $X^{2}(2)=13.89, p<.001$. Forty-three percent $(n=50)$ of the practitioners who stated they strongly agreed or agreed with the statement that they enjoyed the company of animals said they would be interested (or "probably" interested) in participating in AAT. Moreover, only $9 \%$ of those who did not enjoy the company of animals said they would be interested.

Another chi-square test was performed to determine the relationship between therapists' contact with cats (question 11) and whether they would be interested in participating in an AAT program (question 17). Significance was found at $X^{2}(4)=12.24, p<.016$ and it was found that forty-six percent $(n=26)$ of practitioners who stated they often or very often had contact with cats responded that they would participate in an AAT program (or "probably"). Moreover, fortyseven percent of respondents $(n=30)$ who indicated they never or very seldom had contact with a cat stated they would be interested in participating in an AAT program if they had more information about it.

A chi-square test was also performed to determine the relationship between one's contact with dogs (question 10) and their interest in participating in an AAT program (question 17). No significance was found at an alpha level of $.05, X^{2}(4)=8.52, p<.074$.

Another test compared the relationship between the therapists' contact with cats and whether they believe that AAT is a valid treatment modality to be used by OTs; no significance was found, $X^{2}(4)=8.53, p<.074$. Furthermore, no relationship was found $X^{2}(4)=8.17, p<.085$, when looking at the relationship 
between contact with dogs and whether therapists believed AAT is a valid treatment modality.

Another cross tabulation was performed look at the relationship between therapists' participation in AAT (question 16) and whether they think it is a valid treatment modality. Significance was found $X^{2}(2)=12.09, p<.002$, eighty-five percent of respondents $(n=30)$ who indicated that had participated in an animal assisted therapy program also stated they strongly agreed or agreed with the statement that AAT is a valid treatment modality to be used by OTs.

Research question 5: to what extent are OTs' facilities involved in pet visitation programs?

In survey question number 14 , therapists were asked if their facility currently has a pet visitation program in place. Ninety-five practitioners (55.6\%) indicated that their facility does not have a program, sixty $(33.5 \%)$ indicated that they did have a program in place. Moreover, eight therapists $(4.5 \%)$ stated that they did not have a program, but they thought their facility would be more interested if they knew more about it. 


\section{Chapter V}

\section{Discussion}

The purpose of this study was to survey occupational therapists about their attitudes and interests in animal assisted therapy as a treatment modality. The results of this study are discussed in the sections below. Are occupational therapists interested in learning more about AAT as a treatment modality

It was found that most of the practitioners would be interested in learning more about animal assisted therapy. Only $7 \%$ did not want to learn more. Therefore, it is important to educate them about AAT and its uses and benefits. This can be accomplished through more research, more seminars on AAT, studies published in OT-related journals, and media awareness.

These findings are similar to the findings of Funk (1997), who surveyed occupational therapists' interest in therapeutic horseback riding as a treatment modality. This study found that nearly half of the respondents were interested in learning more about therapeutic horseback riding (THR). Furthermore, a similar number indicated "they weren't particularly interested [in learning more about THR], but were willing to read a short article or listen to information on THR" (Funk, 1997). From this study, a recommendation was made to educate current and future OT practitioners about the principles and benefits of THR. AAT as a treatment modality used by occupational therapists

Most of the respondents indicated that they felt AAT is a valid treatment modality. While this may be reflective of the OT population, it could 
also be an artifact of volunteer bias. That is, people who responded to the survey were more inclined to believe in AAT's validity than those who did not return the survey. Nevertheless, apparently the OTs that returned this survey did believe that AAT is a valid treatment modality. So, one must question why occupational therapists don't use it more in the treatment of their patients.

The most common answer to the question "why would you not be interested in participating in an AAT program," was "I feel I don't know enough about it". So, this again points out the need for more research, seminars, and studies published in OT-related journals in order to make therapists more knowledgeable about AAT.

Additionally, the second most common answer to question number 18 $(17.3 \%)$ was reimbursement issues. This may have been affected by the timing of the survey. Therapists received the survey in February of 1999, and at that time changes in the Medicare reimbursement system had recently been established. Importantly, the most common facility that the respondents worked at was a skilled nursing home $(32 ; 17.9 \%)$. A new payment system had been implemented in skilled nursing facilities. These facilities were no longer being reimbursed by Medicare on a retrospective basis based on reasonable costs for ancillary services such as occupational therapy.

Under the Balanced Budget Act of 1997, a prospective payment system (PPS) was instituted, whereas facilities would be reimbursed based on a 
pre-set per diem rate for services. The American Occupational Therapy

Association (1999) concluded,

"The prospective per diem payment system will put the responsibility on SNF [Skilled Nursing Facility] administrators to determine medical needs and then allocate resources to meet those needs. This will be a more complex and potentially more restrictive decision-making process.

Therapists employed by SNFs may be called upon to make decisions about how to manage a limited amount of resources for therapy..."

(AOTA, 1999)

Evidently, many therapists reiterated the concerns of the AOTA about the changes in the reimbursement system. One therapist stated her concern, "the new PPS guidelines no longer allow set up or preparation time to be billable for Medicare A patients. Only direct treatment can be billable. This would limit time consuming pet prep[aration]." Another therapist working in a skilled nursing facility voiced a similar concern, "in the current environment of PPS, there is little time to develop programs. Productivity demands have increased while quality is not to be compromised. It is very stressful now with all the changes. There is so much to learn about PPS, little/no time is left to develop a new program..."

Furthermore, it was found that there was a relationship between therapists' participation in AAT and whether they think it was a valid treatment modality. Those therapists who indicated that had participated in an animal assisted therapy program also stated they strongly agreed or agreed with the statement that AAT is a valid treatment modality to be used by OTs. Importantly, 
this finding demonstrates that once therapists have used AAT, then they will continue to use it. Since it was found that most OTs do not participate in AAT because they feel they don't know enough about it, education is the key to getting OTs interested and knowledgeable about the subject. This knowledge might compel them to participate in AAT programs. Once OTs have used AAT they are more likely to promote its validity and get more therapists involved, therefore expanding the use of AAT.

Patient goals addressed by animal assisted therapy

The most common selected goals included: increase social interaction, sensory stimulation, and increase the display of emotions, whereas the least commonly selected goals included: increase range of motion, increase balance, and strengthening. It is noted that the goals most commonly selected were in the realm of mental health, whereas the least mentioned goals were more physicallybased goals. Apparently therapists see the psychological and social benefits of AAT, but give less consideration to the physical/biological aspects.

These findings are in agreement with the biopsychosocial model, developed by Anne Mosey, an occupational therapist. This model considers an individual as one who suffers from the effects of injury and illness. Moreover, it states that health is more than the absence of disease and emphasizes the interaction between the body, the mind, and the environment of an individual (Miller \& Walker, 1993). Psychological and sociological factors are viewed just as important as the biological factors (Reed \& Sanderson, 1992). Animal Assisted Therapy can be seen through the biopsychosocial model, as it has 
numerous biological, psychological, and sociological effects. Many studies have been done to look at the social and psychological benefits of AAT (Fick, 1993; Taylor et al., 1993; Holcomb \& Meacham, 1989; Adams, 1997; Redefer \& Goodman, 1989; Gonski, 1985; Granger, 1998; Heindl, 1996). More research is needed to evaluate the efficacy of AAT in improving patients' physically-based problems such as balance, strength, and range of motion.

The findings of this survey are also in agreement with Lisa Kongable's study of nursing staff's attitudes about the use of a pet dog among patients with Alzheimer's disease (1990). This study found that nurses ascribed the following benefits of pet visitation: increasing self-esteem, giving patients a "sense of control over their environment", increasing social skills, sensory stimulation, and relaxation.

Furthermore, a survey of administrators in California and South Dakota nursing facilities found (Darrah, 1996) the most common benefit of pet visitation was sensory stimulation. Social interaction, stress reduction, and companionship were the next most commonly reported therapeutic benefits. Interest in participating in animal assisted therapy programs

A relationship was found between therapists' contact with cats and whether they would be interested in participating in an AAT program. Almost half of the practitioners who stated they had contact with cats responded that they would participate in an AAT program (or "probably"). This is understandable, as you would expect that someone who has more contact with cats would be more interested in participating in an animal assisted therapy program. 
However, a chi-square test was also performed to determine the relationship between one's contact with dogs and their interest in participating in an AAT program, and no significance was found. This result is surprising due to the fact that one would expect people who have contact with dogs would be more likely to state that they would be interested in participating in an AAT program. Additionally, this contradicts the findings of Crowly-Robinson \& Blackshaw (1998), where they studied nursing staff members' attitudes toward a pet visitation program in a Veteran's home. They found that staff members who liked dogs favor having a pet visitation program more than those who did not like dogs. To what extent are OTs' facilities involved in pet visitation programs

Thirty-three percent of the respondents indicated that they did have a pet visitation program in place. In comparison, only eleven percent indicated that their facility has an animal assisted therapy program in place. This finding is in agreement with Behling's (1990) survey of animal programs in skilled and intermediate care facilities located in Illinois. This study found that, "ninety-one percent of the facilities that responded allowing nonscheduled animal visits, 57.1 percent having regularly scheduled animal visitation programs, and less than 13 percent having organized animal assisted therapy programs" (Behling, 1990).

Summary

In summary, this study investigated the attitudes and interests of occupational therapists in animal assisted therapy as a treatment modality. The results of this study reveal that a majority of therapists felt AAT is a valid treatment modality. Additionally, OTs that were not involved in AAT did want to 
learn more about it, as they stated the number one reason why they would not be interested in participating in an AAT program was that they felt they do not know enough about it. The major finding from this survey is that to increase the use of AAT, it is essential to educate OTs about the uses and benefits of AAT, as well as reimbursement issues. This can best be accomplished through more research, continuing education programs on $A A T$, publications in professional journals, and media awareness.

It was also found that therapists' most commonly selected patient goals were in the realm of mental health, whereas the least mentioned goals are more physically based goals. Apparently, therapists saw the psychological and social benefits of AAT, but gave less consideration to the physical/biological aspects. More research is needed to evaluate the efficacy of AAT in improving patients' physically based problems such as balance, strength, and range of motion. 
Adams, D.L. (1997). Animal-assisted enhancement of speech therapy: a case study. Anthrozoos, 10(1), 53-56.

American Occupational Therapy Association. (1999, October 1). The Balanced Budget Act of 1997: Skilled Nursing Facilities - Changes in Payment System and Billing Requirements. [WWW document]. URL: http://www.aota.org/featured/area4/links/link7.html

Andrysco, R.M. Pet facilitated therapy in a retirement nursing care community. Research presentation at the International Conference on the Human/Companion Animal Bond, Oct. 5-7, 1981. In: Cusack, O. \& Smith, E. (1984). Pets and the elderly: The therapeutic bond. New York: The Haworth Press.

Arkow, Phil. (Ed.). (1989). The loving bond: Companion animals in the helping professions. Saratoga: R \& E Publishers, Inc.

Beck, A.M., Seraydarian, L., \& Hunter, G.F. (1986). Use of animals in the rehabilitation of psychiatric inpatients. Psychological Reports, 58, 1986.

Behling, R.J. (1990). Animal programs and animal-assisted therapy in skilled and intermediate care facilities in Illinois. Unpublished doctoral dissertation.

Bernard, S. (1995). Animal Assisted Therapy: A guide for health care professionals and volunteers. Tyler: Tyler Press.

Baun M.M. et al. (1984). Psysiological effects of human/companion animal bonding. Nursing Research, 33(3), 126-129.

Brickel, C.M. (1984). Depression in the nursing home: A pilot study using pet-facilitated psychotherapy. In R.K Anderson. B.L. Hart, \& L.A. Hart (Eds.), The Pet Connection. Minneapolis.

Brickel, C.M. (1979). The therapeutic roles of cat mascots with a hospitalbased geriatric population: a staff survey. The Gerontologist, 19(4), 368-372. Brickel (1984).

Bruce, M.A. \& Borg, B. (1993). Psychosocial Occupational Therapy. Thorofare, NJ: SLACK Incorporated.

Casey, H.M. (1996). A survey of occupational therapists using petfacilitated therapy. Home Health Care Manage Practice, 8(4), 10-17. 
Crowley-Robinson, P. \& Blackshaw, J.K. (1998). Nursing home staffs' empathy for a missing therapy dog, their attitudes to animal-assisted therapy programs and suitable dog breeds. Anthrozoos, 11(2), 101-104.

Cusack, O. \& Smith, E. (1984). Pets and the elderly: The therapeutic bond. New York: The Haworth Press.

Darrah, J.P. (1996). A pilot survey of animal-facilitated therapy in Southern California and South Dakota nursing homes. Occupational Therapy International, 3(2), 105-121.

Edwards, G. S. (1998, November 18). A physician's view of animal assisted therapy [WWW document]. URL:

http://home.sprynet.com/sprynet/bouvweb/ aatlettr.htm

Fick, K. (1993). The influence of an animal on social interactions of nursing home residents in a group setting. The American Journal of Occupational Therapy, 47, 529-534.

Friedman, E. et al. (1980). Animal companions and one-year survival of patients after discharge from a coronary care unit. Public Health Reports, 95(4), 307-312.

Funk, M. (1997). A survey of occupational therapy practitioners' interest in therapeutic horseback riding as a treatment modality. Unpublished master's thesis, Florida International University, Miami, Florida.

Gonski, Y. (1985). The therapeutic utilization of canines in a child welfare setting. Child \& Adolescent Social Work Journal, 2(2), 93-105.

Granger, B.P., Kogan, L., Fitchett, J, \& Helmer, K. (1998). A humananimal intervention team approach to animal-assisted therapy. Anthrozoos, 11(3), 172-176.

Harris, M.D., Rinehart, J.M., \& Gerstman, J. (1993). Animal-assisted therapy for the homebound elderly. Holistic Nursing Practice, 8(1), 27-37.

Heindl, B.A. (1996). The effectiveness of pet therapy as an intervention in a community-based children's day treatment program. Dissertation Abstracts International, 57, 1501.

Hines, L. \& Bustad, L. (1986). Historical perspectives on human-animal interactions. Phi Kappa Phi Journal, Spring, 4-9. 
Holcomb, R. \& Meacham, M. (1989). Effectiveness of an animal-assisted therapy program in an inpatient psychiatric unit. Anthrozoos, 2(4), 259-264.

Howell-Newman, K. \& Goldman, R.L. (1993). Marketing animal facilitated therapy. Health Marketing Quarterly, 11(1/2), 77-98.

Hume, L. (1998, November 13). Animal Facilitation in Occupational and Physical Therapy Sessions. [WWW document]. URL: http://www.rehabnet.com/aft/aftotpt.htm

lannuzzi, D. \& Rowan, A.N. (1990). Ethical issues in animal-assisted therapy programs. Anthrozoos, IV(3), 154-163.

Jenkins, J.L. (1986). Physiological effects of petting a companion animal. Psychological Reports, 58, 21-22.

Johansson, C. (1998). 'Llama love' therapy. OT Week, 12(36), 12-13.

Katcher, A.H. (1985). Physiologic and behavioral responses to companion animals. Veterinary Clinics of North American Small Animal Practice, 15(2), 403410.

Kielhofner, G. \& Burke, J.P. (1980). A model of human occupation. Part one: Conceptual framework and content. American Journal of Occupational Therapy, 34, 572-581.

Kielhofher, G. (1997). Conceptual Foundations of Occupational Therapy. Philadelphia: F.A. Davis Company.

Kongable, L.G., Stolley, J.M., \& Buckwalter, K.C. (1990). Pet therapy for Alzheimer's patients: A survey. The Journal of Long-Term Care Administration, $\underline{18}(3), 17-21$.

Kongable, L.G., Buckwalter, K.C., \& Stolley, J.M. (1989). The effects of pet therapy on the social behavior of institutionalized Alzheimer's clients. Archives of Psychiatric Nursing, 3(4), 191-198.

Kranz, J.M. \& Schaaf, S. (1989). Nursing-home staff attitudes toward a pet visitation program. Journal of the American Animal Hospital Association, 25 , 409-417.

Limond, J.A., Bradshaw, J.W.S., Cormack, K.F.M. (1997). Behavior of children with learning disabilities interacting with a therapy dog. Anthrozoos, 10(2/3), 84-89. 
Miller, R. J., \& Walker, K. F. (1993). Perspectives on theory for the practice of Occupational Therapy. Gaithersburg, MD: Aspen Publishers.

NAHRA (1995a). NAHRA Guide. Denver: Author. In: Funk, M. (1997). A survey of occupational therapy practitioners' interest in therapeutic horseback riding as a treatment modality. Unpublished master's thesis, Florida International University, Miami, Florida.

Nathanson, D.E., de Castro, D, Friend, H. McMahon, M. (1997). Effectiveness of short-term dolphin-assisted therapy for children with severe disabilities. Anthrozoos, 10 (2/3), 90-100.

Perelle, I.B. \& Granville, D.A. (1998, November 8). Assessment of the Effectiveness of a pet facilitated therapy program in a nursing home setting. [WWW document]. URL: http://arrs.envirolink.org/psyeta/sa/sa1.1/perelle.html.

Redefer, L.A. \& Goodman, J.F. (1989). Brief Report: Pet-facilitated therapy with autistic children. Journal of Autism and Developmental Disorders, 19 (3), 461-466.

Reed, K. L., \& Sanderson, S. N. (1992). Concepts of Occupational Therapy ( ${ }^{\text {rd }}$ ed.). Baltimore, MD: Williams \& Wilkins.

Robb, S.S, Boyd, M. \& Pristash, C.L. (1980). A wine bottle, a plant and a puppy: catalysts for social behavior. Journal of Gerontological Nursing, 6(12) In: Cusack, O. \& Smith, E. (1984). Pets and the elderly: The therapeutic bond. New York: The Haworth Press.

Robb, S.S. (1981). "Pilot study of pet-dog therapy for elderly people in long term care" Unpublished report. In: Cusack, O. \& Smith, E. (1984). Pets and the elderly: The therapeutic bond. New York: The Haworth Press.

Rowell, M.C. (1990). Creature comfort: Animals as therapists. California Pharmacist, 37(10), 37-40.

Serpell, J.A. (1990). Evidence for long term effects of pet ownership on human health. Waltham Symposium 20: Pets, Benefits \& Practice. First European Congress of the British Small Animal Veterinary Association. Cheltenham, England.

Taylor, E. et al. (1993). Effect of animals on eye contact and vocalizations of elderly residents in a long term care facility. Physical and Occupational Therapy in Geriatrics, 11(4), 61-71. 
Thompson, M., Kennedy, R. \& Igou, S. "Pets as socializing agents with chronic psychiatric patients." Research presentation at the International Conference on the Human/Companion animal Bond, Oct. 507, 1981. In: Cusack, O. \& Smith, E. (1984). Pets and the elderly: The therapeutic bond. New York:

The Haworth Press.

Walsh et al. (1995). The effects of a 'pets as therapy' dog on persons with dementia in a psychiatric ward. Australian Occupational Therapy Journal, 42, 161-166.

Westbrook and Katz (1985). Pet-facilitated therapy programs: planning considerations and risk-minimization procedures. Clinical Management in Physical Therapy, 5(6), 26-28.

Zisselman et al. (1996) A pet therapy intervention with geriatric psychiatry inpatients. The American Journal of Occupational Therapy, 50, 47-51. 
APPENDICES 


\section{Survey Cover Letter}

11065 NW $39^{\text {th }}$ Street

Apartment 302

Sunrise, FL 33351

Dear Occupational Therapist:

I am a graduate student in occupational therapy at Florida International University in Miami, FL. As a requirement for my master's degree, I am currently working on my thesis. My study addresses the attitudes and interests of occupational therapists in animal assisted therapy.

Enclosed is a brief survey that should take no more than five to ten minutes to complete. I have also enclosed a self-addressed, stamped envelope for your convenience. The survey includes demographic information as well as questions regarding animal assisted therapy. Please note that the survey questions are printed on both sides of the paper in an effort to conserve our natural resources. All respondents will remain anonymous and the results will be recorded as group data. Completion of this survey indicates that you agree to participate in this study.

Your cooperation in completing and returning this survey as soon as possible would be greatly appreciated. Thank you very much.

Sincerely,

Lori S. Buckley, OTS 
1. Please indicate your gender.
a. Male
b. Female

2. Please indicate your age.

3. Please indicate the highest level of education that you have achieved.
a. Bachelor's degree in occupational therapy
b. Entry level master's degree in occupational therapy
c. Advanced Master's degree in occupational therapy
d. Master's degree in another field (please specify)
e. Doctorate in occupational therapy
f. Doctorate in another field (please specify)
g. Other (please specify)

4. How many years have you been practicing as an OTR?
a. Less than 1 year
d. 7-10 years
b. 1-2 years
e. 11-16 years
c. 3-6 years
f. 17 years +

5. What city and state are you currently practicing in?
a. city, state
b. I am not currently practicing

6. What is your current area of primary practice in occupational therapy? (please circle the ONE that you work in most)
a. School system
b. Developmental disabilities
c. Other pediatric areas
d. Physical disabilities
e. Gerontology
f. Home health

g. Administration and management

h. Mental health

i. Work hardening

j. Education

k. Other (please specify)

7. At what kind of facility do you primarily work? (please circle only one)
a. Day care program
b. Home health agency
c. Hospice
d. Independent living center, group home, or a residential care facility
e. General hospital - rehab unit
f. General hospital - other areas
g. Pediatric hospital
h. Psychiatric hospital
i. Outpatient clinic (freestanding)
j. Physician's office
k. Private practice

1. Public health agency

m. Rehabilitation Center or hospital

n. Research facility

o. Retirement or senior center

p. School system (including private schools)

q. Sheltered workshop/supported employment

r. Skilled nursing facility or intermediate care facility

s. University/College

t. Voluntary Agency

u. Other (please specify) 
8. Please indicate how long you have been working at this particular facility.
a. Less than 1 year
d. 7-10 years
b. 1-2 years
e. 11-16 years
c. 3-6 years
f. 17 years +

9. Please indicate the common diagnoses of the patients you see at your facility (please circle all that apply):
a. AIDS
b. Alzheimer's disease
c. Amputation
d. Arthritis
e. Autism
f. Cancer
g. Cerebral Palsy
h. Cerebral vascular accident (CVA)
i. Dementia
j. Depression
k. Developmental disabilities
I. Hearing impairments
m. Mentally impaired
n. Multiple Sclerosis (MS)

o. Muscular dystrophy

p. Orthopedic conditions

q. Parkinson's disease

r. Traumatic brain injury (TBI)

s. Visual impairments

t. Schizophrenia

u. Spina Bifida

v. Spinal Cord injury

w. Other (please specify)

10. How often do you have any contact with a dog?
a. Never
d. Sometimes
b. Very seldom
e. Often
c. Seldom
f. Very often

11. How often do you have any contact with a cat?
a. Never
b. Very seldom
d. Sometimes
c. Seldom
e. Often
f. Very often

12. Do you agree with this statement? I enjoy the company of animals such as dogs or cats.
a. Strongly agree
d. Disagree
b. Agree
e. Strongly disagree
c. Neither agree or disagree

13. What is your present knowledge/awareness of animal assisted therapy (AAT)? (Please circle all that apply)
a. I have participated in animal assisted therapy with
b. I have read about it in journals, books, newsletters, etc.
(Please specify type of animal)
c. I have heard about it through word of mouth
d. I have attended a structured presentation on AAT (i.e. in-service, continuing education)
e. I heard about AAT in my school curriculum
f. I have never heard of AAT
g. Other (please specify) 
14. Pet visitation is when animals accompany their owners to a facility to visit the patients. The main goal of animal visitation is socialization. It is often used by the nursing profession. Does your facility currently have a pet visitation program in place?
a. Yes
b. No, but we are trying to institute one
c. No, but I think my facility would be interested in having one if we knew more about AAT
d. No, but we have had a program in the past
e. No

15. Animal Assisted Therapy (AAT) is defined as the use of animals as a therapeutic modality in order to facilitate the rehabilitation of patients with chronic or acute illnesses. Animal Assisted Therapy is utilized by many professionals, such as Occupational Therapists, Physical Therapists, Speech Therapists, and Psychiatrists. The animals are used as a treatment modality in goal directed treatment sessions. Does your facility currently have an animal assisted therapy program in place using dogs and cats?
a. Yes
b. No, but we are trying to institute one
c. No, but I think my facility would be interested in having one if we knew more about AAT
d. No, but we have had a program in the past
e. No

16. Have you ever participated in an animal assisted therapy program?
a. Yes (please skip \#17-18 if you circled yes)
b. No

17. If you've never participated in an animal assisted therapy program, would you be interested in participating in one?
a. Yes, definitely
c. I might if I had more information about it
b. Probably
d. Probably not
e. No

18. Why would you not be interested in participating in an animal assisted therapy program? (please circle all that apply)
a. I don't have the time
b. I'm concerned about the possible legal issues
c. I feel I don't know enough about it
d. I don't want to work with animals
e. I'm concerned about the safety of the patients
f. I'm concerned about the safety of the animals
g. I'm concerned about cleanliness
h. I'm concerned about pet transmitted diseases
i. Reimbursement issues
j. I am allergic

k. I don't think the patients would want to work with animals

I. I am interested but my facility doesn't support it

m. I don't believe AAT is effective

n. Other (please specify) 
19. Do you agree with this statement? I would be interested in learning more about animal assisted therapy as a treatment modality.
a. Strongly agree
d. Disagree
b. Agree
e. Strongly disagree
c. Neither agree nor disagree

20. Do you agree with this statement? Animal assisted therapy is a valid treatment modality to be used by occupational therapists.
a. Strongly agree
d. Disagree
b. Agree
c. Neither agree nor disagree
e. Strongly disagree
f. I don't know

21. What patient goals do you think animal assisted therapy addresses (please check all that apply):
a. Increase range of motion
b. Increase social interaction
c. Increase balance
d. Increase coordination
e. Decrease tone
f. Strengthening
g. Speech/communication skills
h. Increase the display of emotions
i. Sensory stimulation
j. Decrease depressive symptoms
k. Increase reality orientation

I. Increase concentration

m. Decrease anxiety

n. To facilitate patient-therapist interaction

o. None of the above

p. I haven't heard of AAT

q. Other (please specify)

22. Please list any comments/suggestions 